\title{
DIFFERENTIAL IDENTITIES IN PRIME RINGS
}

\author{
ABDELKARIM BOUA and AHMED Y. ABDELWANIS
}

\begin{abstract}
Let $\mathcal{R}$ be a prime ring with center $Z(\mathcal{R})$ and $\alpha, \beta: \mathcal{R} \rightarrow \mathcal{R}$ be automorphisms. This paper is divided into two parts. The first tackles the notions of (generalized) skew derivations on $\mathcal{R}$, as the subject of the present study, several characterization theorems concerning commutativity of prime rings are obtained and an example proving the necessity of the primeness hypothesis of $\mathcal{R}$ is given. The second part of the paper tackles the notions of symmetric Jordan bi $(\alpha, \beta)$-derivations. In addition, the researchers illustrated that for a prime ring with $\operatorname{char}(\mathcal{R}) \neq 2$, every symmetric Jordan bi $(\alpha, \alpha)$-derivation $D$ of $\mathcal{R}$ is a symmetric bi $(\alpha, \alpha)$-derivation.
\end{abstract}

MSC 2010. 16W25, $16 \mathrm{U} 80$.

Key words. Prime rings, skew derivations, symmetric Jordan bi $(\alpha, \beta)$-derivation.

\section{REFERENCES}

[1] M. Ashraf, A. Asma and A. Shakir, On Lie ideals and generalized $(\theta, \phi)$-derivations in prime rings, Comm. Algebra, 32 (2004), 2977-2985 .

[2] M. Ashraf and N. Rehman, On commutativity of rings with derivation, Results Math., 42 (2002), 3-8.

[3] M. Ashraf, N.-ur-Rehman and M. Rahman, On generalize derivations and commutativity of rings, Internat. J. Math., Game Theory, and Algebra, 18 (2008), 19-24.

[4] M. Bresar and J. Vukman, Jordan derivations on prime rings, Bull. Aust. Math. Soc., 37 (1988), 321-322.

[5] M. Bresar and J. Vukman, On left derivations and related mappings, Proc. Am. Math. Soc., 10 (1990), 7-16.

[6] Y. Çeven and Z. Çiloğlu, On symmetric Jordan and Jordan left bi-derivations of prime rings, Afr. Mat., 29 (2018), 689-698.

[7] I.N. Herstein, Jordan derivations of prime rings, Proc. Am. Math. Soc., 8 (1957), 11041110 .

[8] G. Maksa, A remark on symmetric biadditive functions having nonnegative diagonalization, Glas. Mat., 15 (1980), 279-282.

[9] E. Posner, Commuting derivations in prime rings, Proc. Amer. Math. Soc., 8 (1957), $1093-1100$.

The authors thank the referee for his helpful comments and suggestions.

DOI: $10.24193 /$ mathcluj.2021.2.06 
Received January 23, 2020

Accepted August 31, 2020
Sidi Mohammed Ben Abdellah University Polydisciplinary Faculty

LSI, Taza, Morocco

E-mail: abdelkarimboua@yahoo.fr

Cairo University

Faculty of Science

Department of Mathematics

Giza, Egypt

E-mail: ayunis@sci.cu.edu.eg 